\title{
Integration Issues on Combining Feature Technology and Life Cycle Assessment
}

\author{
H.E. Otto, K.G. Mueller and F. Kimura \\ Dept. of Precision Engineering, The University of Tokyo \\ Bunkyo-ku, Hongo 7-3-1, Tokyo 113-8656, JAPAN \\ tel. $+81-3-5841-6495-f a x .+81-3-3812-8849$ \\ e-mail:[otto,kmueller,kimura]@cim.pe.u-tokyo.ac.jp
}

Abstract Within recent research trends and an increased interest in targeting not only product modeling but product life cycle modeling as a whole, for feature technology used to support and enhance mainly computer-aided design and computer-aided manufacturing, new dimensions of requirements arise. Further demands on higher automation, more flexibility, enhanced man-machine interaction and increased built-in knowledge of application systems, require sophisticated (system) interfaces and perhaps even local re-design of feature frameworks currently developed. To combine and relate data from both a product model and a life cycle inventory data base, currently several (in most cases manual, non optimized) methods were employed. However, they usually all ignore the potential of predefined structures and soft technologies now-a-days integrated within a product model. Within the scope of the work described in this paper, the focus will be on studying and discussing the approach to extend a feature model in order to explicitly utilize feature technology within product life cycle assessment. In particular methods and structures for two assessment approaches namely discrete and continuous life cycle assessment will be introduced and discussed.

Keywords Feature technology, model and framework integration, product design, life cycle inventory parameters, life cycle assessment

\section{INTRODUCTION}

Work on feature technology as it evolved in the past two decades grew from its origin to identify part regions of interest for manufacturing of products to a powerful technology with many frameworks and system architectures developed as well as implemented. However, with recent research trends and an increased interest in targeting not only product modeling but product life cycle modeling (LCM) as a whole, for feature technology used to support and enhance mainly computer-aided design and computer-aided manufacturing, new dimensions of requirements arise. Feature technology is a powerful tool that at certain 
representation levels, can be successfully used to capture the relationship of shape and functionality in many ways. An important aspect if one recalls the most laborintensive work within life cycle assessment (LCA), which is to determine the life cycle inventory ( $\mathrm{LCI})$. On the other hand, approaching automation of extracting LCI data elements from already available digital models such as product models and CAD models, requires efficient interfacing between life cycle assessment software and these models. However, within this context as outlined, to properly utilize functionality of feature technology on a broad base, framework and entity concepts developed up to now, need to be extended. Within the scope of the work described in this paper, the focus will be on studying and discussing the approach to extend a feature model in order to explicitly utilize feature technology within product life cycle assessment. In particular methods and structures for two assessment approaches namely discrete and continuous life cycle assessment will be introduced and discussed.

\section{FEATURE TECHNOLOGY AND LCA}

\subsection{Background and problems}

Early work on features was done during the late 1970's and early 1980's within problems of identifying regions of part geometry important to manufacturing. After half a decade of untiring efforts, when feature technology left its initial embryonic state, integration of feature models and geometrical models was targeted with the basic goal to integrate different processes such as manufacturing and design. Although, from the very beginning, the basic idea of features was to associate functional information with shape information, early feature frameworks and implementations of feature systems displayed a low degree of integration between a feature model and a geometrical model. These individual approaches were focused on either a more function/meaning related approach $[4,14]$ or a more geometry related approach $[16,6]$.

Besides integration and consistency problems partially mastered in frameworks developed in the late 1980's and early 1990's [22,21,23,17], successful modeling of relationships and control of feature properties across features as well as across different feature classes represent a further important milestone in the historical development of feature technology (cf. [24]). Extensions of feature modeling domains not only towards geometry in particular [5,1], but also towards further applications within assembly modeling [18,9] and product life cycle modeling $[13,19,12]$ are further indicators of efforts to translate into practice and integrate the potential of FT within today's rapidly evolving fields of product life cycle assessment and modeling.

The first approaches, to what today is called life cycle assessment, were developed in the 1960 's. The studies of that time dealt mainly with energy issues [2], which was related to the general interest in the scarcity of raw materials and fossil fuels. Formalized LCA tools developed in the USA were mainly based on raw materials and energy analysis, such as the REPA method by [10]. The 
development of LCA procedures began in Europe in the early 1980's, especially in Switzerland, where the Ministry of the Environment initiated research lead to the development of the Critical Volumes Method, and the Ecopoint Method [7]. Several European research groups began publishing on LCA methods in the 1990's, often supported by national agencies (e.g. CML classification method in the Netherlands by Heijungs et al. [8]), Environmental Priority Strategies (EPS) method in Sweden [26]). Related methods, such as the KEA method [28] and Material Intensity Per Service-unit (MIPS) [25] were also developed in Europe. In the recent past the International Standards Organization has issued a framework for conducting LCA's [11].

The difficulty of LCA is not based on the complexity of the methodology, but on the complexity of problem. Each single step is very simple and can be understood easily but the often thousands of steps required to assemble a complete LCI make a accurate LCA extremely difficult and expensive. To give an impression of the scale associated with compiling an LCI, Bretz [3] estimated that an analyst may require some $10^{4}$ to $10^{5}$ or more numerical data elements for a complex product which will consist of thousands of unit processes.

Although, as can be seen from the brief survey given above, progress in various different directions within the fields of feature technology and life cycle assessment has been achieved, essential concepts and structures that support an efficient integration of both from the viewpoint of information/knowledge modeling and processing within the entire product life cycle as discussed above, are still missing.

\subsection{Scope and objectives}

As pointed our earlier, in life cycle assessment the most labor-intensive work is to determine the life cycle inventory. This is also because it is the most data-intensive part and extremely difficult to automate. A natural, straightforward solution to this problem would be to automate the extraction of LCI data elements from CAD drawings to make LCA a viable analysis tool during the design process. However, this can only be achieved by efficiently interfacing product model software and life cycle assessment software.

An example of a previous approach is an experimental assessment software which consists of evaluation modules integrated with a commercial CAD system, extended for handling form features on a limited base. This prototype system integrates information on possible manufacturing methods, production costs and eco-toxicological data. It is primarily aimed at optimizing the manufacturing method of the embodiment design, so that the emissions and costs due to manufacturing are minimized. The system extracts geometrical and feature information from both the geometrical model and the feature model and so evaluates manufacturing costs and energy requirements during the manufacturing stage [15]. The disadvantage of this implementation is that it is based on an inflexible architecture, resulting in a 'closed system', thus there is no possibility of interfacing this system to exchange the product's pre-use inventory data with existing LCA software, which would allow a more complete life cycle assessment. 
An alternative, better structured and more flexible approach introduced and discussed in the next section is to utilize the inventory information already available in product and process models for determining the LCI in an automated fashion. This requires the linking and integration of different models and data bases on a system and module based firm footing. To overcome shortcomings of related work discussed above, system component interfacing and information integration are realized by employing a true feature technology supported open system architecture.

However, within this approach two different directions can be chosen, depending on the type of LCA, i.e. the time range and frequency, LCI parameters are updated and eventually computation of LCA is carried out during computer-aided design sessions. Within the scope of the work described, the focus will be on evaluating continuous LCA, a progressive type of computation and discrete LCA, an aposteriori type of computation.

\section{APPROACH}

\subsection{Outline and general framework}

To integrate different system modules and models as outlined earlier, one requires system components with an open system architecture that provides an application programming interface (API) for each relevant model to be accessed by a LCA module. For example as shown in Figure 1, this requires at least one API for the process model and also at least two for the product model, providing a means to connect the geometrical model and the feature model to individual LCA modules.

Any local or explicit access to models other than from within its own modeler must not be allowed, to guarantee a clean and modular architecture where access to individual models is only permitted through a dedicated API. Especially in the case of geometrical models, due to their central role within a product model, a welldesigned, attribute supporting API is indispensable. 


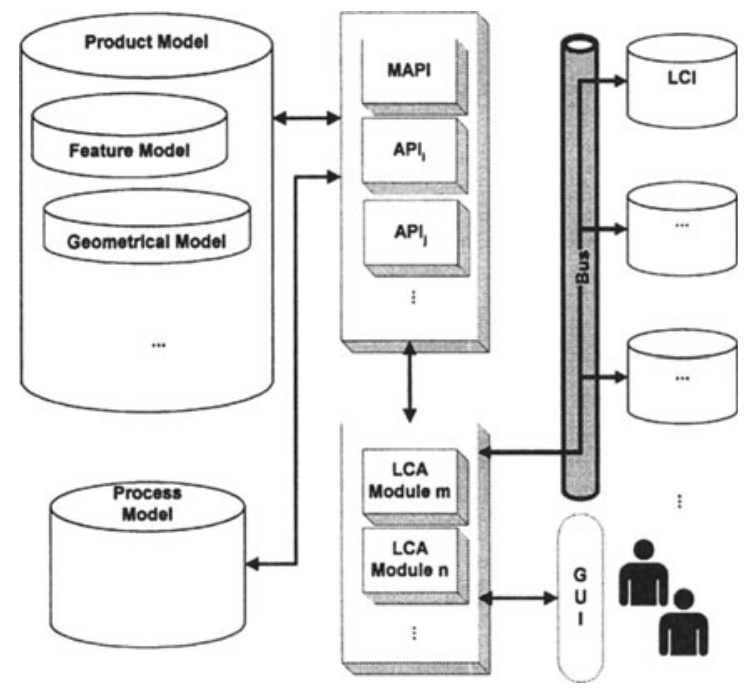

Figure 1 Overview of the system architecture

Unfortunately in practice there are still many commercially available CAD systems and geometrical modelers in use, which do not support any of these characteristics. Due to the fairly wide range of data types, the context of API parameters needs to be set a-priori by individual LCA modules. For example, in the case of the API for the feature model, measurement units of parameters such as feature dimensions or feature volume need to be fixed, to guarantee the proper interpretation and integration of data from different sources. Attributed parameters also help to keep the interfacing API transparent, since the number of functions and parameters required can be kept small, while only the range of individual interface components is modeled with appropriate attribute values. A design that keeps the API flexible, while fixing the data context on a dynamic base.

Another aspect of flexibility is the modular architecture for the API of a feature model. Due to the nature of features being both application dependent and user extensible, the feature API needs to have a modular internal interface that can be extended according to newly created feature types and feature attributes. These modular feature API (see MAPI in Figure 1) also operating on the basis of attributed parameters, are then flexible enough, to provide on a feature-based ground efficient access to product and process information.

\subsection{Discrete life cycle assessment}

From a computing system and data structure point of view, as pointed out earlier, there are two different approaches, to support LCA, which will be introduced in the following and discussed in detail in the next section. The main difference of these 
two approaches lies in the method and degree, how LCI parameters and LCA models are integrated with other product relevant models utilizing feature technology. LCI parameters are seen as those parameters that relate to the LCI directly or indirectly, many of which are already included in some explicit or implicit form in a product model, but only need to be linked in a correct and efficient manner to determine the LCI. In Figure 2 a selection of those parameters is given. Note, for illustration purposes three levels of detail are distinguished, i.e., the product level, the part level / assembly level and the feature level.

One approach to integrate these parameters with product information would be to provide a set of integrated LCA modules that contain partial LCI / LCA models which can be linked to product information and a LCI database, to externally compute as well as store LCA results. Since this approach is, from the viewpoint of computer-aided product design, somewhat discrete being applied only from time to time when a design solution seems to be worth being analyzed in respect to its product life cycle qualities, it is named discrete life cycle assessment (dLCA). Within ILCA the entire data retrieval and access, data analysis and computation of relevant LCI parameters is specified and carried out within integrated LCA modules. 


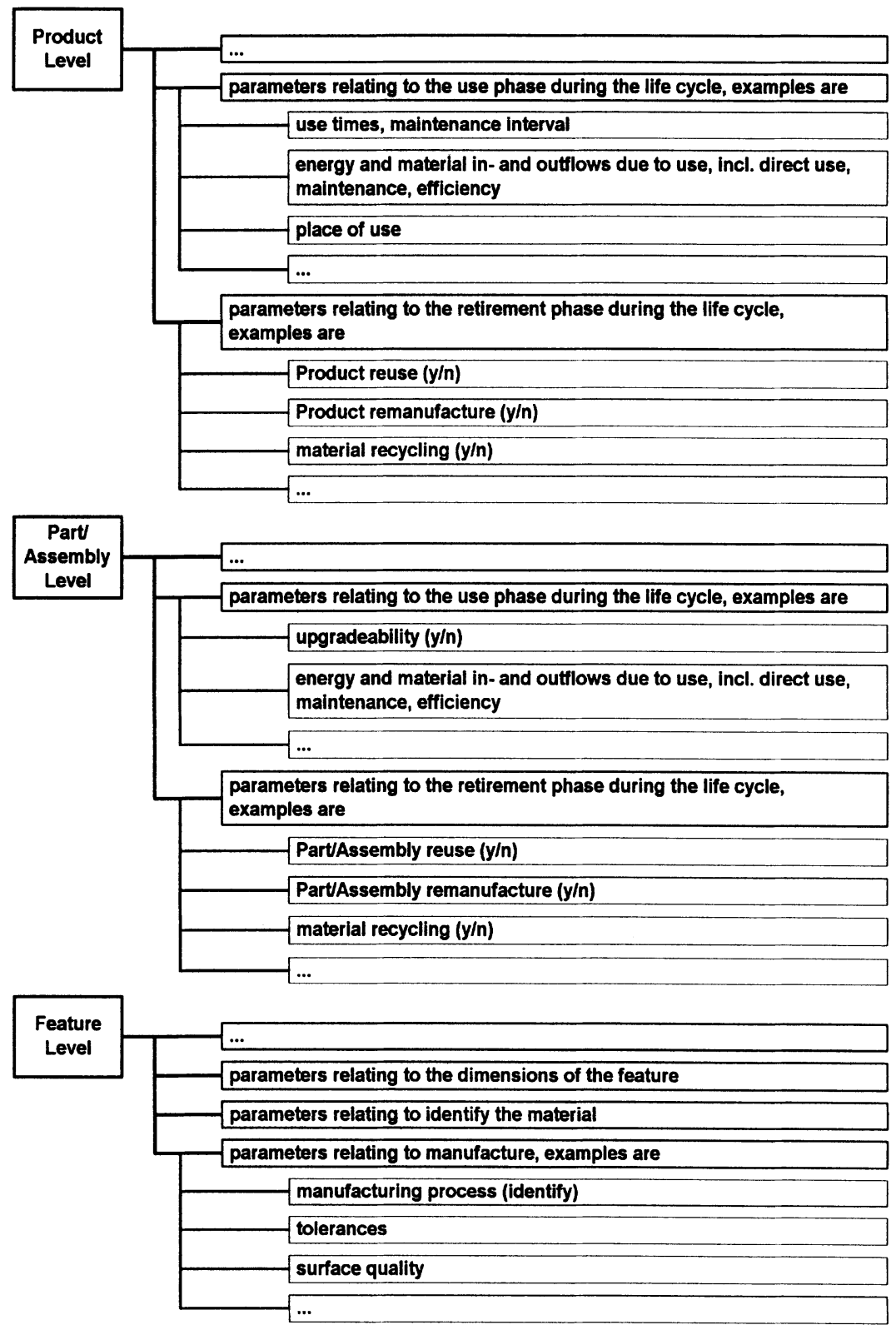

Figure 2 A selection of LCI relevant parameters 


\subsection{Continuous life cycle assessment}

An alternative approach would be to explicitly integrate LCI relevant parameters, partial LCA models and results of LCA within models of a product. Within such a system architecture, during different design stages of a product, each change in any model immediately would be triggering a recalculation and consequently an updating of all LCI parameters and LCA results as well. Within this approach, besides LCI relevant parameters as shown in Figure 2, further parameters, containing LCA results of individual entities such as features, parts, assemblies or even a complete product, need to be included. A representative selection of those parameters may be a set as shown in Figure 3.

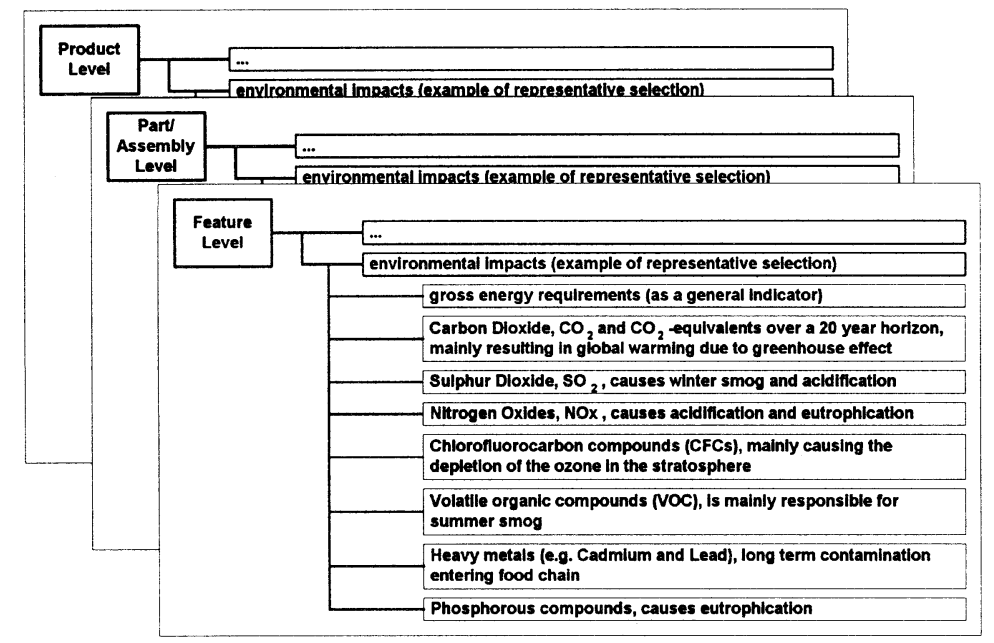

Figure 3 Representative selection of parameters containing LCA results on environmental impacts

Within this approach, all product data, LCI parameters and LCA results to be computed are explicitly interrelated on a real-time base. Since any design change is triggering an update of all relevant entities connected across all models being included in the framework, LCA is, from a computer-aided product design point of view, carried out on a progressive, continuous base, thus resulting in the name continuous life cycle assessment (cLCA). Within cLCA, the majority of data access and retrieval, data analysis and computation of relevant LCI data is specified and carried out internally in data and computation structures which are - as encapsulated entities - an integrated part in all product related models. LCA modules integrated in the framework as outlined earlier act here mainly as an external support to efficiently interface to further related databases such as the LCI database, etc. 


\section{ASSESSMENT AND EVALUATION}

\subsection{Theoretical aspects}

For the dLCA approach, since computation of the LCA is mostly executed externally, a fast and efficient access to all LCI relevant data is necessary. As can be seen in Figure 2, many LCI parameters at the product level and part / assembly level can be represented with basic numerical values, logical values and a few string values. However, to compute LCA parameters also geometrical and geometry related product data are required. Here integrated feature models represent a great benefit from a data structure point of view. All parameters related to dimensions, manufacturing, local tolerances, surface finish, etc. are already present in a feature model that in turn is integrated with other product model components such as the geometrical model the part / assembly model, etc. Also procedures to access these data are already available within feature-based operators, which only need to be made available to the LCA modules through a MAPI as shown in Figure 1. For the cLCA approach, from a data structure point of view, the feature model represents even an essential part of the product model, for without features, it would be, due to efficiency reasons (as will be shown later), very difficult to pursue this approach. Since besides relevant LCI parameters all sets of computed LCA parameters (cf. again Figure 3) are included in an extended product model, one needs a set of basic elements of a product, where internal LCA parameter computation can be associated with. Here features represent a natural, almost atomic base, where geometry related details of all parts of a product were stored and maintained in a manner consistent to all other, non-geometry related product data.

From a computation structure point of view, requirements and benefits of the two approaches are quite different. In the case of dLCA, the specification of partial LCA models and LCA parameter computation resides entirely within integrated LCA modules, which also contain structures and descriptions on how to access an LCI database. Since partial LCA models are externally specified within LCA modules and computation of LCA parameters is asynchronuous to changes in a product model, no special requirements regarding computation or parameter updating are necessary. In the case of CLCA, both results of computed LCA parameters and specification of their computation reside (on a real time base) within an extended product model. In other words, the functionality of LCA modules within dLCA as described earlier needs to be encapsulated within extended entities at different levels of a product model. Moreover, sophisticated mechanisms are required that guarantee a synchronized bi-directional bottom-up as well as top-down data flow among all integrated LCI and LCA parameters along all model levels. They are necessary to provide for a consistent parameter updating each time a design change has been committed in the product model.

Taking a brief look at the computational expense of both approaches, it comes at no surprise, that the cLCA approach is fairly expensive in terms of data throughput and computation required. Since cLCA needs to re-compute and update all related parameters, any time a change in the product model is affecting at least one of them, computational expenses increase tremendously with the model size. It is 
estimated that with completion of each design phase described in [20] as conceptual design, embodiment design and detail design, the data volume is exponentially increasing (see Figure 4) in a similar manner as costs do when changing design parameters. The cost associated with changes to the design increases by a factor of 10 for each design stage and changes in the manufacturing stage can cost more than 1,000 times the amount that would have been required if the decision would have been reached during the concept stage [27]. It has to be realized that a LCA carried out at the later stages can be more accurate due to the higher product definition, but the increased data throughput and computation required also increases the effort for the LCA. This means that when an assessment of the LC leads to changes in the design, then it is more cost-effective to both assess and change early on during the design process.

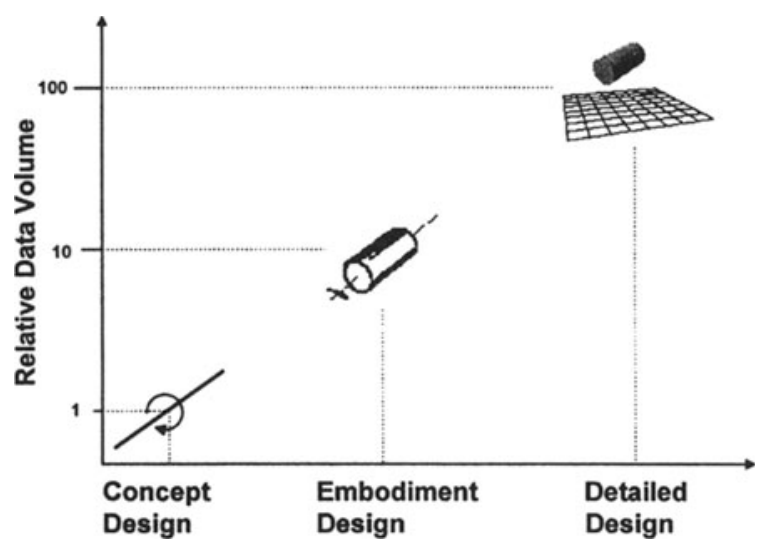

Figure 4 Estimated order of magnitude of product data required in each design phase

Although dLCA is much cheaper in absolute terms of both data throughput and computation, we estimate the order of magnitude of expense to retrieve and access all parameters before executing another LCA calculation within ILCA is in average similar to one sequence of updating and data forwarding within CLCA. Here FT helps to significantly reduce through its well structured model and efficient access to geometry related data the otherwise cumbersome access to LCI relevant product parameters. An aspect that is also relevant for the dLCA, though compared to cLCA less dominant, since actual LCA is done much less frequently than within cLCA. In general, if frequency of computing LCA parameters increases within each approach, FT's role as outlined above becomes a dominant factor to reduce substantially computational expense for both approaches.

From a computer-aided product design point of view, the scope possible of supporting individual design phases as mentioned earlier depends for both approaches on the data structure and models used within a system. Referring to each design phase, dLCA and cLCA are limited by the smallest, most abstract but complete entity available, which in the framework as described, is the feature. In 
other words, within both approaches, limits are lined out by the feature model used. In general one can assume, since features can only be abstracted as far as there is actually at least concrete geometry of a spatial region of interest available, currently LCA in both forms discrete and continuous can be supported only from embodiment design on, not earlier. Although, LCA is most effective the earlier it is applied during product design, at the time of writing, feature models supporting conceptual design are not known to the authors.

\subsection{Practical aspects}

From the practical viewpoint of implementation dLCA is a straight forward approach that can be pursued without major obstacles, assuming an open system architecture as outlined in Figure 1 is given. A modular system component structure and APIs for all relevant models provide an ideal, well-structured environment to implement dLCA without heavy requirements on the programming environment itself, though attributed structures, a sound typing scheme and if possible, objectoriented approaches should be supported. In the case of cLCA, implementation requirements are heavier. Besides an attribute-like structure extension to allow for storing additional LCI and LCA parameters in an extended model, the entire computation of the latter needs to be somehow encapsulated. Moreover at run time, a bi-directional data flow between all LCI and LCA parameters needs to be established and synchronized. In terms of supporting implementation, FT provides only moderate help for dLCA. Besides reducing specification / programming expenses by providing through an API already available feature-based operators for navigating and accessing geometry related data, there is not much additional support FT can provide for dLCA. Different is the case for CLCA. If feature models are not included in a product model, similar constructs or support structures need to be implemented first. It would be quite expensive from a computation point of view to retrieve and compute all geometry related LCI parameters by utilizing a geometrical model and a part / assembly model only.

Finally to complete the assessment and evaluation of practical aspects, the scope of detail of carrying out LCA shall be discussed. In the case of dLCA, it is relatively easy to explicitly control the scope of computation related to e.g. each life cycle phase of a product or a specific part of the product. Simply by setting the focus of retrieval and access of relevant LCI parameters to certain product model levels and respective entities, thus selectively limiting the data input for the LCA, computation can be directed in individual LCA modules towards individual parts and life cycle phases as demanded by a designer. A task that is explicitly supported by FT in respect to all structural and computational aspects as discussed earlier. However, in the case of cLCA this is quite different. Due to the encapsulated structures that prevail throughout all portions of a product model deep down to the feature model, explicit control of the scope of computation regarding the range of LCI parameters used for the LCA is not possible. For example, exclusive local LCA of a part of a product, regarding its pre-use and post-use is not possible within cLCA without additional changes of components in the system architecture as outlined earlier. Therefore, current LCA methods as carried out in practice now a 
days, computing LCA parameters once for an entire product for all three life cycle phases and afterwards computing only new values of critical parts regarding their pre-use and post-use phase - neglecting assumed small changes incurred in the usephase - while product design proceeds, are difficult to support with CLCA. To support such methods, additional data structures and computation structures are required, to allow for parameter selection and data stream re-direction of once during implementation specified and encapsulated structures related to LCI and LCA. Here FT may provide some structural help only, if it can adopt to these requirements within its model and operators.

\section{CONCLUSIONS}

LCA is becoming more important as an assessment tool within the design process. To support interfacing of computer-aided design and LCA, in a first attempt, the problem of extending the architecture and product data related models of current systems utilizing FT has been targeted. In particular methods and structures for two assessment approaches, i.e. dLCA and cLCA, haven been introduced and discussed. Considering the current state-of-the-art of CAD systems and computer hardware available today, requirements of dLCA clearly present a reasonable match regarding data throughput, data structures and computational power required. Also from both a theoretical and a practical point of view, until more LCI data with higher precision and reliability become available, a less precise dLCA with respect to small changes during design - reflected also in modifications of a product model - seems better to be in balance with current LCI databases than the costly CLCA. On the other hand, if new generations of software and hardware platforms significantly increase in efficiency, quality and power, it might be not far fetched, to assume that CLCA shall be the proper choice to base future research and development on.

\section{REFERENCES}

[1] Au, C.K. and Yuen, M.M.F. (2000) A semantic feature language for sculptured object modeling, Computer-Aided Design, 32, 1, 63-74.

[2] Boustead, I. (1995) Life cycle assessment: An overview, Energy World: The Magazin of the Institute of Energy, 230, 7-11.

[3] Bretz, R. (1998) SETAC LCA workgroup: Data availability and data quality, International Journal of LCA, 3, 3, 121-123.

[4] Descotte, Y. and Latombe, J.C. (1984) GARI: An Expert System for Process Planning, in: Solid Modeling by Computers (Picket and Boyse, eds.), Plenum Press, New York, USA.

[5] Fontana, M., Giannini, F. and Meirana, M. (1999) Free form feature taxonomy, Computer Graphics Forum, 18, 3, 107-118.

[6] Gossard, D.C., Zuffante, R.P. and Sakurai, H. (1988) Representing Dimensions, Tolerances, and Features in MCAE Systems, IEEE Computer Graphics \& Applications, 8, 2, 51-59. 
[7] Habersatter, K. (1991) Report No. 132: Ecobalance of Packaging Materials State of 1990, Swiss Federal Office of the Environment, Forests and Landscape (BUWAL), Berne, Switzerland.

[8] Heijungs, R., Guinee, J., Huppes, G., Lankreijer, R. M., de Haes, H. A. U., Sleeswijk, A. W., Ansems, A. M. M., Eggels, A. M. M., Duin, R. V. and de Goede, H. P. (1992) Environmental life cycle assessment of products, guidelines and backgrounds, Technical report, Centre of Environmental Sciences (CML), Leiden University, The Netherlands.

[9] van Holland, W. and Bronsvoort W.F. (2000) Assembly features in modeling and planning, Robotics and Computer Integrated Manufacturing, 16, 4, 277-294.

[10] Hunt, R., Sellers, J. D. and Franklin, W. (1992) Resource and environmental profile analysis: A life cycle environmental assessment for products and procedures, Environmental Impact Assessment Review, 12, 245-269.

[11] ISO 14040 ff. (1997) DIN EN ISO 14040 ff: Umweltmanagement - Ökobilanz, Beuth, Berlin, Germany.

[12] Krause F.-L., Kind, Ch. and Martini, K. (1997) Application of Feature Technology in a Disassembly-Oriented Information Technology Infrastructure, in: Proc. 4-th CIRP International Seminar on Life Cycle Engineering, June 26-27, Berlin, Germany, 345355.

[13] Krause F.-L., Stiehl, Ch. and Martini, K. (1998) New Applications for Feature Modeling, in: Proc. Israel-Korea Bi-National Conference on new Themes in Computerized Geometrical Modeling, February 18-19, Tel Aviv, Israel, 1-9.

[14] Luby, S.C., Dixon, J.R. and Simmons, M.K. (1986) Creating and Using a Features Database, Computers in Mechanical Engineering, 5, 3, 25-33.

[15] Müller, D.H. and Oestermann, K. (1997) Simultaneous consideration of ecological and economical parameters in early stages of product design, in: Proc. International Conference On Engineering Design, August 19-21, Tampere, Finland, Vol.1, 337-342.

[16] Ostrowski, M.C. (1987) Feature-based Design using Constructive Solid Geometry, Internal Report, General Electric Corporate Research and Development, Schenectady, New York, USA.

[17] Otto, H.E., Mandorli, F., Cugini, U. and Kimura, F. (1994) Domain-Oriented Semantics For Feature Modeling Based On TAE Structures Using Conditional Attributed Rewriting Systems, in: Proc. ASME International Design Engineering and Computers in Engineering Conference, September 12-14, Minneapolis, MN, 13-27.

[18] Otto, H.E., Kimura, F. and Mandorli, F. (1998) Support of Disassembly / Reassembly Evaluation within Total Life Cycle Modeling Through Feature Neighborhoods, in: Proc. of ASME International Design Engineering and Computers in Engineering Conference, September 13-16, Atlanta, GA.

[19] Otto, H.E. and Kimura, F. (1998) Towards a framework for unmitigated integration of feature technology within life cycle modeling, in: Proc. of JSPE 1998 Annual Conference, March 18-20, Kawasaki, Kanagawa, Japan.

[20] Pahl, G. and Beitz, W. (1996) Engineering Design: A Systematic Approach, SpringerVerlag, New York.

[21] Pratt M.J. (1989) A Hybrid Feature-Based Modelling System, in: Preprints International Symposium on Advanced Geometric Modelling For Engineering Applications, November 8-10, Berlin, Germany, 177-189.

[22] Rossignac, J.R., Borrel, P. and Nackman, L.R. (1988) Interactive design with sequences of parametrized transformations, Research Report RC 13740, IBM Research Division, Thomas J. Watson Research Center, Yorktown Heights, New York, USA. 
[23] Shah, J.J. and Rogers, M.T. (1989) Feature Based Modeling Shell: Design and Implementation, in: Proc. ASME Computers in Engineering Conference, July/August 30-3, Anaheim, CA, 255-261.

[24] Shah J.J. (1991) Assessment of features technology, Computer-Aided Design, 23, 5, $331-343$.

[25] Schmidt-Bleek, F. (1997) Wieviel Umwelt braucht der Mensch? Faktor 10 - das Maß für ökologisches Wirtschaften, dtv, Taschenbuchausgabe, Germany.

[26] Steen, B. and Ryding, S. O. (1994) Valuation of environmental impacts within the EPS-system, in: SETAC: Integrating Impact Assessment Into LCA, SETAC-Europe, 155-160.

[27] VDI-Richtlinie 2210 (1975) Datenverarbeitung in der Konstruktion; Analyse des Konstruktionsprozesses im Hinblick auf den EDV-Einsatz, VDI-Verlag, Düsseldorf, Germany.

[28] VDI-Richtlinie 4600 (1995) Kumulierter Energieaufwand - Begriffe, Definitionen, Berechnungsmethoden (Entwurf), VDI-Verlag, Düsseldorf, Germany. 